\title{
Astronomical Plate Archives as Astrophysical Facility and Tool to Study Microquasars
}

\author{
René Hudec \\ Astronomical Institute of the Academy of Sciences of the Czech Republic \\ E-mail: rene.hudec@gmail.com \\ Vojtěch Šimon \\ Astronomical Institute of the Academy of Sciences of the Czech Republic \\ E-mail: vojtech.simon@gmail.com
}

\section{Lukáš Hudec}

Astronomical Institute of the Academy of Sciences of the Czech Republic

E-mail: matfyzakeseznam.com

\begin{abstract}
There are about 3 million astronomical photographic plates around the globe, representing an important data source for various aspects of astrophysics. The main advantage is the large time coverage of 100 years or even more, as well as good sampling. The recent digitization efforts, together with the development of dedicated software, enables for the first time effective data mining and data analyzes by powerful computers with these archives. We will present the recent status of such efforts, and then we will focus on the astrophysical aspects with emphasis of application in high-energy astrophysics (HE) (where many HE sources do have optical counterparts accessible by plates) including microquasars. The importance of the astronomical data archives as additional data source for such analyzes will be discussed. Examples of microquasars investigated on the astronomical plates will be presented.
\end{abstract}

VII Microquasar Workshop: Microquasars and Beyond

September 1 - 5, 2008

Foca, Izmir, Turkey 


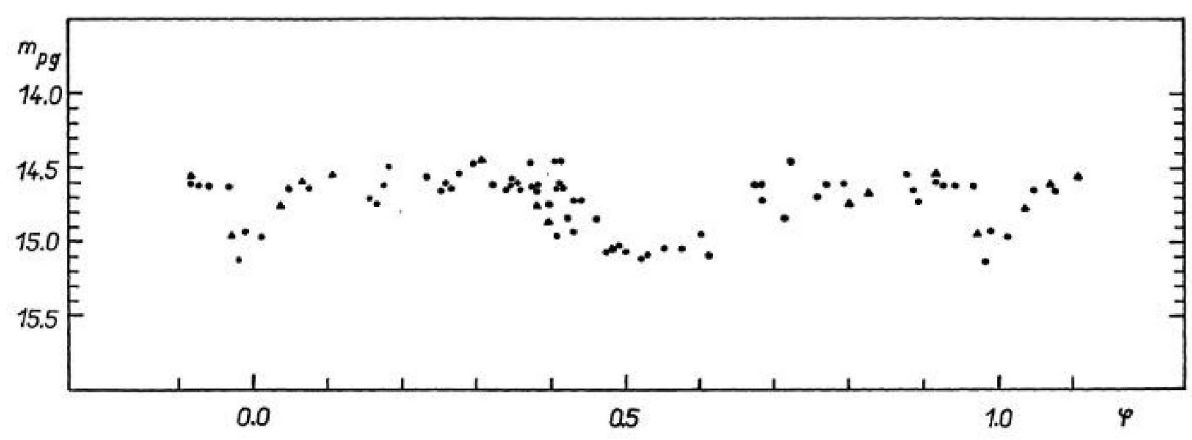

Figure 1: The optical orbital modulation of HZ Her, the optical counterpart of Her X-1, during its rare inactive state. Circles denote the observations between JD 2428630 and JD 2429789. Triangles mark the data between JD 2427543 and JD 2427657 (Hudec \& Wenzel 1976).

\section{Introduction}

The study of the long-term activity of astrophysical objects is an important but not easy task. In many cases we need very-long term optical light curves covering more than one decade, and in some cases even more than several decades, as some duty cycles in some types of astrophysical targets can be so long (e.g. in binary blazars). For the time being, the astronomical plate archives represent a suitable source, as they can provide up to several thousands of photometric points covering up to 100 years or so.

\section{Astronomical plate archives and microquasars}

The knowledge of long-term activity of known microquasars is still very limited. It is evident that the use of data gained from data mining in astronomical plate archives can be important. In this paper we show a few examples.

In Fig. 1 we show an example of the study of the low-mass X-ray binary Her X-1/HZ Her on the astronomical astrograph plates from the Sonneberg Observatory, showing the behavior of the object during rare inactive state of the system.

More recently, we have started analyzes of selected microquasars on southern archival plates located at the Bamberg Observatory. As an example, the appearance of the microquasar V4641 Sgr both in quiet as well as in flaring state is shown in Fig.2. The preliminary results obtained from these plates confirm the presence of the optical flaring activity (Fig. 7).This is a part of the project performed at the Bamberg Observatory (Figs.3 and 4).

The long-term light curve of the microquasar Sco X-1/V818 Sco based on the Sonneberg sky patrol data shows an interesting feature of the long-term high and low state (Fig.5).

An additional interesting and important case is the possibility to study the spectral features and spectral changes using the astronomical plates taken with the objective prisms, i.e. analyzing the low-dispersion spectra. Here new dedicated algorithms have been recently developed and tested (Fig.6), allowing automated classification of objects as well as searches for the objects with unique spectra and investigations of the spectral changes. 


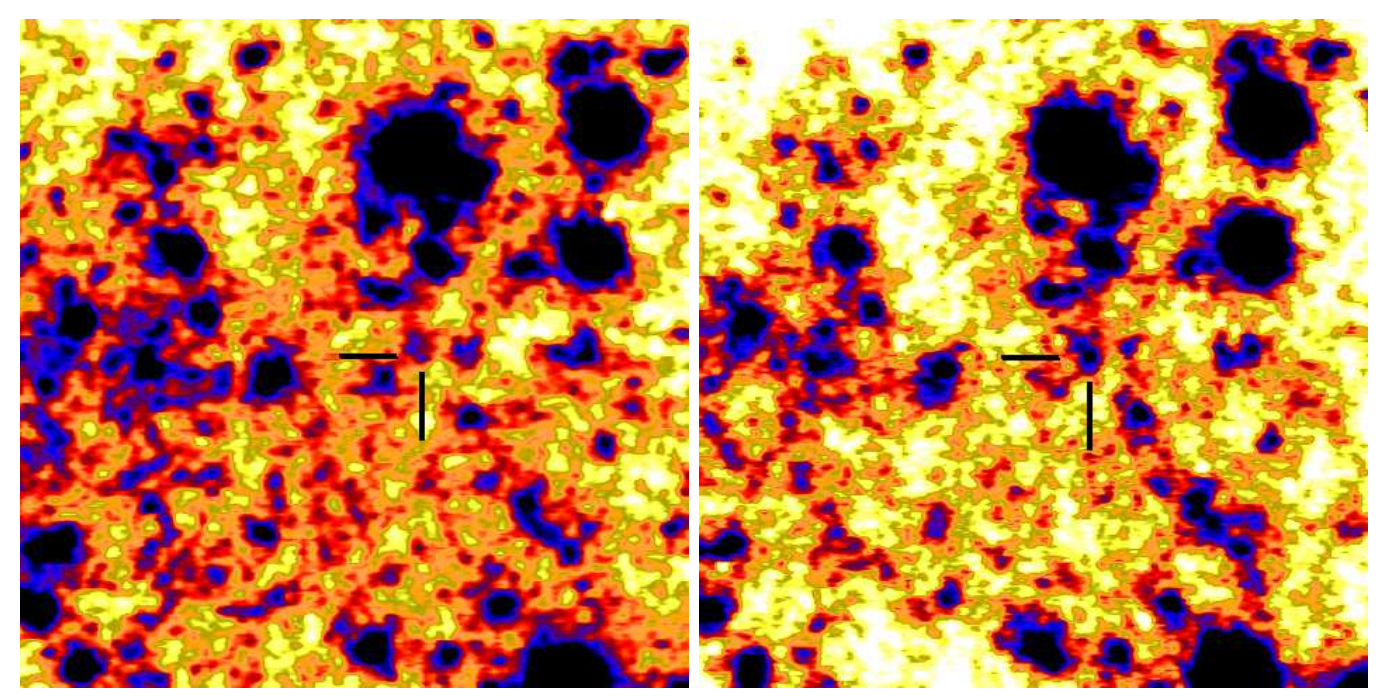

Figure 2: The appearance of the microquasar V4641 Sgr on the digitized sky patrol archival plates from the Bamberg Observatory. The object is shown both in the minimum light (plate SUD 0181, left) as well as in maximum light (plate SUD 8332, right).
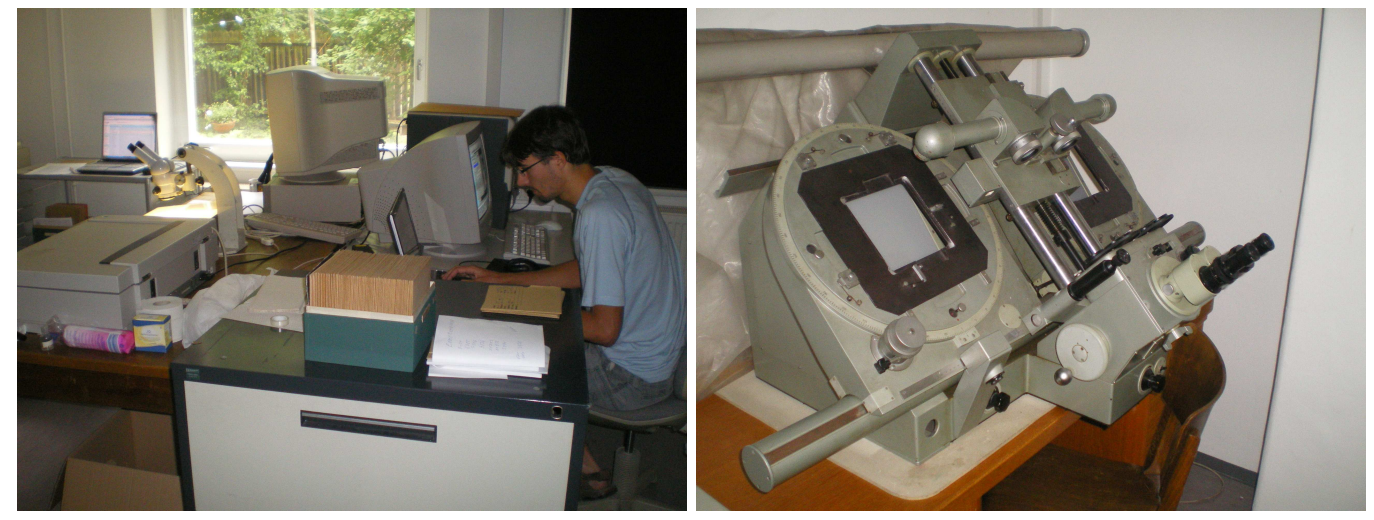

Figure 3: The newly reconstructed laboratory of the astronomical plate archive at the Bamberg Observatory (left) and the Bamberg Observatory blink microscope (right).
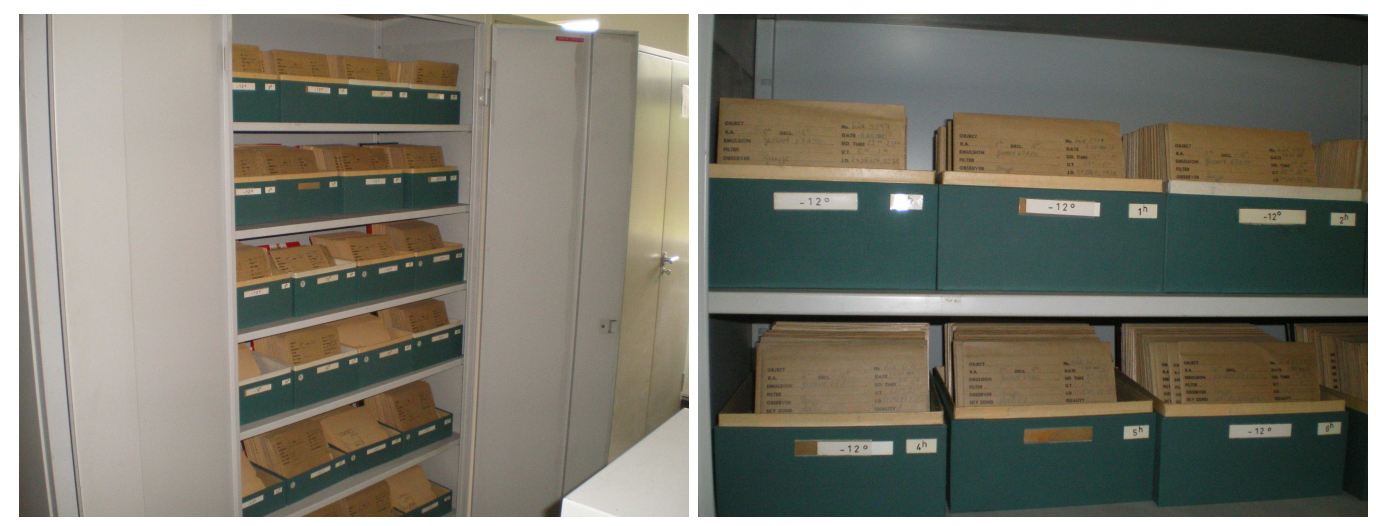

Figure 4: The Bamberg plate archive - one of the plate cabinets (left) and the arrangement of the plates in the cabinets in Bamberg (right). 


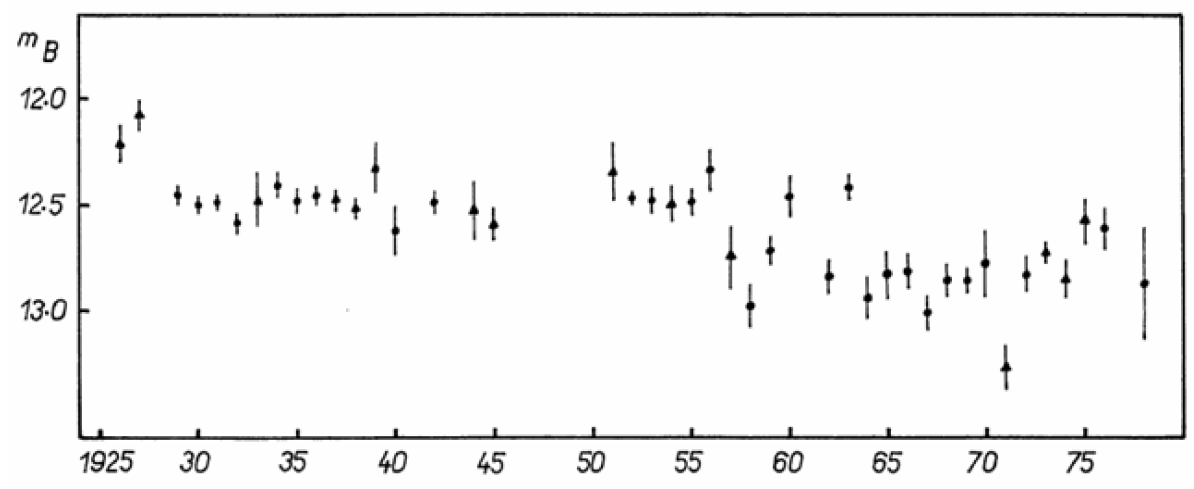

Figure 5: Long-term optical light evolution of V818 Sco, the optical counterpart of Sco X-1. Annular mean values are given. Error bars show the standard deviation of the mean. Time axis is displayed in years (Hudec 1981).

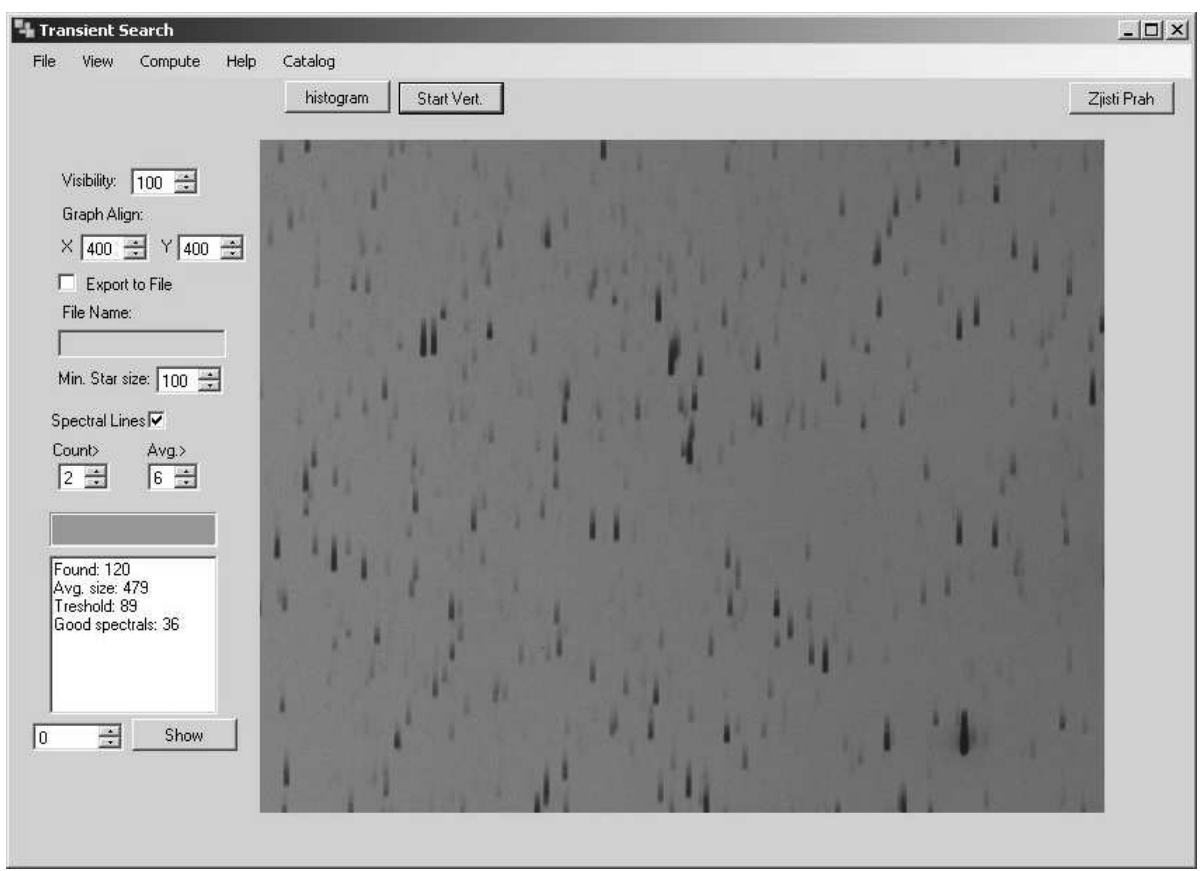

Figure 6: The automated analysis of a part of the digitized astronomical spectral Schmidt plate from the Sonneberg Observatory plate archive.

\section{Conclusion}

The astronomical plate archives represent a suitable data source for the analyzes of the longterm activity of many categories of astrophysical sources including microquasars. The novel computer-based methods including plate scanning and evaluation by dedicated sophisticated software play a major role here.

\section{Acknowledgments}

We acknowledge the grant 205/08/1207 provided by the Grant Agency of the Czech Republic. 


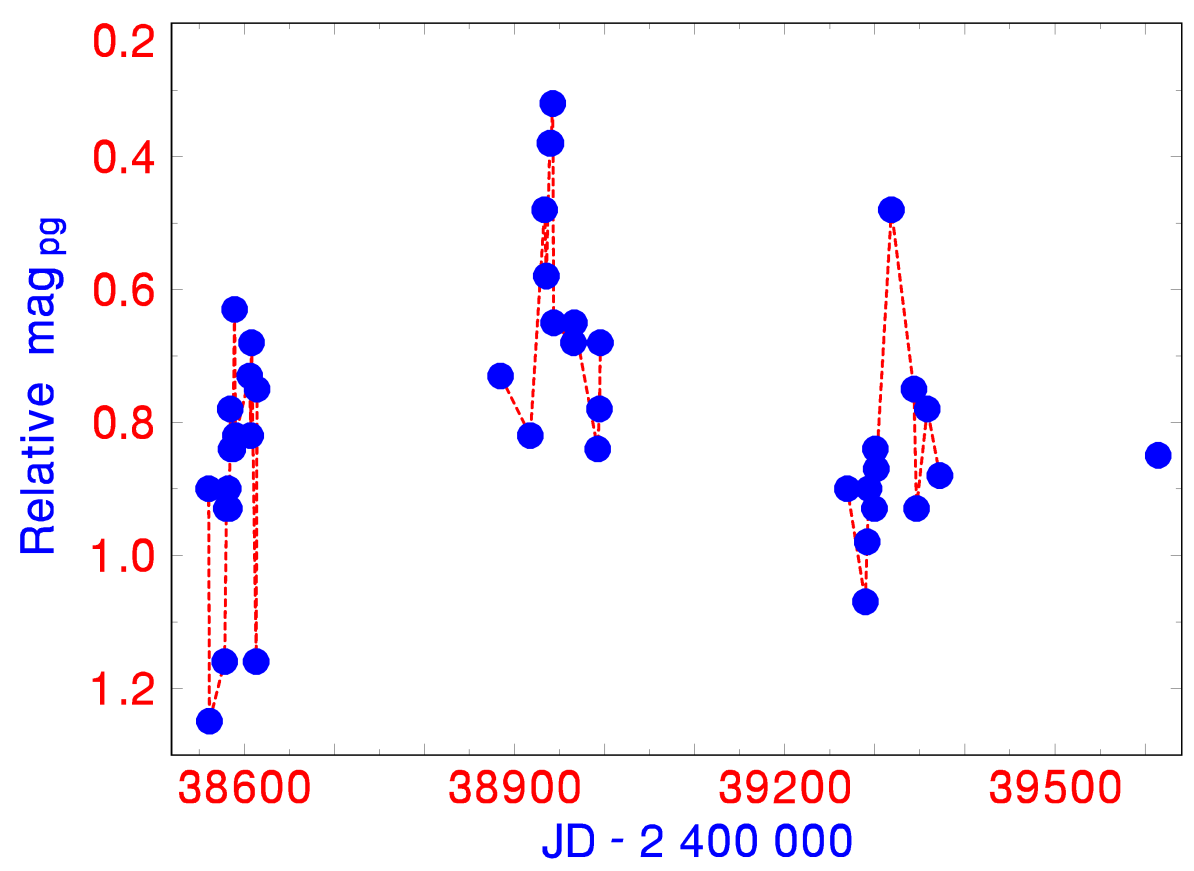

Figure 7: The optical light curve of the microquasar V4641 Sgr on the blue-band sensitive photographic plates from the archive of the Bamberg Observatory (1964-1967). The exposure time was 60 min. Typical uncertainty is 0.05 mag. Magnitudes are displayed with respect to the star GSC 0684802768.

Some aspects of the project described here represent a natural continuation of the Czech participation to the ESA INTEGRAL (ESA PECS 98023).

\section{References}

[1] M. A. C. Perryman, in Astrometry in the Age of the Next Generation of Large Telescopes, ASP Conference Series, Vol.338, San Francisco: Astronomical Society of the Pacific, p.3 (2005).

[2] C. Jordi, J. M. Carrasco, in The Future of Photometric, Spectrophotometric and Polarimetric Standardization, ASP Conference Series, Vol.364, San Francisco: Astronomical Society of the Pacific, p.215 (2007).

[3] R. Hudec, W. Wenzel, Observations of HZ Her/Her X-1 on Sonneberg Astrograph Plates, BAICz 27, 325 (1976).

[4] R. Hudec, Optical Properties of X-ray Stars. 2. The Secular Brightness Variations of the X-ray System $V 818$ Sco/Sco X-1, BAICz 32, 108 (1981).

[5] R. Hudec, V. Simon, Specific object studies for cataclysmic variables and related objects, ESA Gaia Reference Code GAIA-C7-TN-AIO-RH-001-1 (2007a).

[6] R. Hudec, V. Simon, Specific object studies for optical counterparts of high energy sources, ESA Gaia Reference Code GAIA-C7-TN-AIO-RH-002-1 (2007b).

[7] L. Hudec, Algorithms for spectral classification of stars, BSc. Thesis, Charles University, Prague (2007). 